\title{
One-dimensional off-chip beam steering and shaping using optical phased arrays on silicon-on-insulator
}

\author{
Karel Van Acoleyen, Student Member, IEEE, Katarzyna Komorowska, Wim Bogaerts, Member, IEEE, \\ and Roel Baets, Fellow, IEEE
}

\begin{abstract}
Optical beam steering can find applications in several domains such as laser scanning, LiDAR (Light Detection And Ranging), wireless data transfer and optical switches and interconnects. As present beam steering approaches use mechanical motion such as moving mirrors or MEMS (MicroElectroMechanical Systems) or molecular movement using liquid crystals, they are usually limited in speed and/or performance. Therefore we have studied the possibilities of the integrated silicon photonics platform in beam steering applications. In this paper, we have investigated a 16 element one-dimensional optical phased array on silicon-on-insulator with a field-of-view of $23^{\circ}$. Using thermooptic phase tuners, we have shown beam steering over the complete field-of-view. By programming the phase tuners as a lens, we have also shown the focusing capabilities of this onedimensional optical phased array. The field-of-view can easily be increased by decreasing the width of the waveguides. This clearly shows the potential of silicon photonics in beam steering and scanning applications.
\end{abstract}

Index Terms-Silicon on insulator technology, grating couplers, beam steering, optical phased arrays, thermooptic effects.

\section{INTRODUCTION}

O PTICAL beam steering has a great potential in diverse applications. Free-space beam steering can on the one hand be used in laser scanning applications such as LiDAR (Light Detection And Ranging), optical wireless links, remote node interrogation ... but can also find applications in the very short range such as in optical switches and optical interconnects. As interconnects are currently becoming a bottleneck in increasing chip speed, this has gained a lot of interest [1].

While beam steering can be done mechanically, this is usually less stable and limited in speed. Optical Phased Arrays (OPAs) provide a way to efficiently steer an optical beam without any mechanical motion, making them insensitive to acceleration. Liquid crystals have received a lot of attention in OPA technology, but suffer from limited steering angle and limited speed. In these systems, the orientation of the liquid

K. Van Acoleyen, K. Komorowska, W. Bogaerts and R. Baets are with the Photonics Research Group, Department of Information Technology (INTEC), Ghent University-imec, 9000 Ghent, Belgium and the Center for Nano- and Biophotonics (NB-Photonics), Ghent University, 9000 Ghent, Belgium, email: karel.vanacoleyen@intec.ugent.be.

K. Van Acoleyen acknowledges the Research Foundation - Flanders (FWO) for a research grant. This work was supported by the Methusalem project Smart Photonic ICs of Ghent University and the IAP project Photonics@be

Copyright (c) 2011 IEEE. Personal use of this material is permitted. However, permission to use this material for any other purposes must be obtained from the IEEE by sending a request to pubs-permissions@ieee.org.

Manuscript received Month ??, 20??; revised Month ??, 20??. crystal induces a change in refractive index and they thus act as a programmable lens that can steer the light [2].

In this paper, we have investigated the use of the silicon photonics platform in beam steering applications. While silicon photonics gives us many integrated functions on-chip, the interface between the outside world is very important. This becomes increasingly difficult when one does not couple to an optical fiber, but directly to free-space, such as in free-spacing interconnecting, sensing or scanning applications. Beam steering on this platform has already been investigated in [3] and [4] where a one- and two-dimensional OPA on silicon-on-insulator (SOI) has been demonstrated, respectively. In the former, the beam could be steered to a small angle as there was only one heater electrode. In the latter, sidelobe levels were high due to the very small fill factor of the OPA. In [5], the same platform has been used with irregularly spaced optical phased arrays for one-dimensional large angle beam steering using thermo-optic phase shifters. This relaxes the $\lambda / 2$ constraint to avoid the different output orders of the optical phased arrays. However the sidelobe level becomes quite high.

Here we present a design that combines both fixed and thermo-optic beam steering. Due to the grating used at the output, light is coupled off-chip and the wavelength can be used to steer the beam in the other dimension. As has been shown in [6], a beam can also be steered in the twodimensional space using wavelength tuning only. By changing the input waveguide of the star coupler, fixed steering is accomplished. Making use of a star coupler will also give us better far-field characteristics as there is a Gaussian envelope across the light emitting elements due to the free propagation region of the star coupler.

The $3 \mathrm{~dB}$ field-of-view (FOV) is limited to about $23^{\circ}$, but can easily be increased by reducing the width of the grating coupler waveguides. A trade-off has to be made between the FOV and the beam width, as the number of addressable spots in free-space depends on the number of radiating elements of the OPA. Using a grating etched on a $450 \mathrm{~nm}$ wide photonic wire, the FOV can become as large as $160^{\circ}$.

We have also demonstrated focusing capabilities of an OPA on SOI. As the OPA is actually a one-dimensional programmable lens, light can be focused above chip in one dimension. This becomes increasingly interesting when one wants to use a photonic integrated circuit (PIC) for sensing applications, such as in on-chip laser Doppler velocimetry [7] 
or Optical Coherence Tomography (OCT) [8]. Light is for example focused on the skin and reflected back in the PIC for further processing. The beam can then be shaped to optimize the link losses.

In the next Section, we will discuss the design and fabrication of the components. In Section III, the measurement setup is discussed. Section IV gives the measurement results. A conclusion is finally formulated in Section V.

\section{DESIGn AND FABrication}

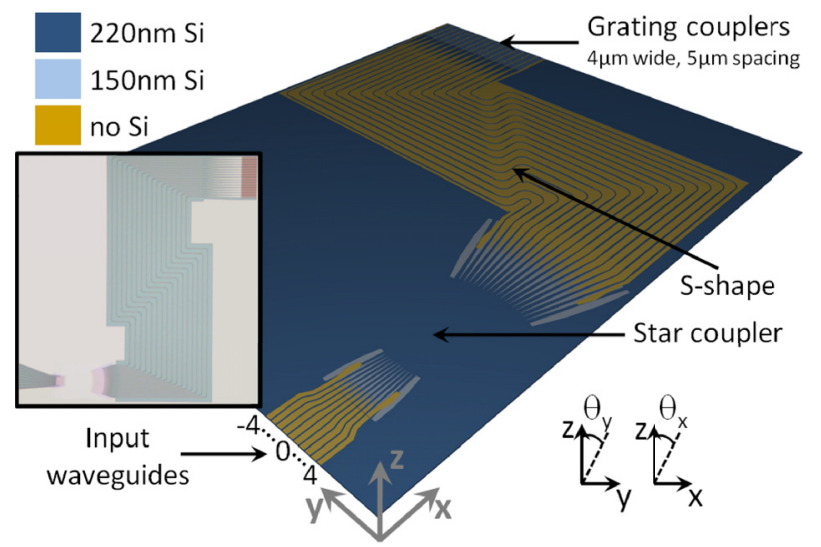

Fig. 1. Virtual fabrication of a one-dimensional OPA on SOI. Light enters through the input waveguides of the star coupler and couples off-chip using grating couplers, on the S-shape heaters are processed (not shown in the Figure) to tune the respective phases of the different grating couplers. The inset shows a microscope image of the fabricated component before heater processing.

The beam steering component shown in Fig. 1 was fabricated in imec, Leuven through ePIXfab (www.epixfab.eu) which uses standard CMOS (Complementary Metal Oxide Semiconductor)-compatible processes to fabricate photonic integrated circuits [9]. An SOI wafer with a $2 \mu \mathrm{m}$ thick buried oxide layer and a 220nm top silicon layer was used. Two deep-UV (UltraViolet) lithography etch steps are then used: one of $220 \mathrm{~nm}$ to pattern the waveguides and the star coupler and one of $70 \mathrm{~nm}$ to pattern the out-coupling gratings and the tapering sections of the star coupler. Using a two-step tapering approach, the insertion loss of the star coupler is reduced to less than $1.5 \mathrm{~dB}[10]$.

The waveguides in the S-shape (Fig. 1) are $800 \mathrm{~nm}$ wide as wider waveguides are more tolerant to fabrication deviations and reduce the corresponding phase errors in the waveguides. At each bend, the waveguide tapers back to a $450 \mathrm{~nm}$ wide single mode wire, which allows very sharp bends of less than $3 \mu \mathrm{m}$, without significant loss. This S-shape ensures all waveguides are in phase and allows us to use a large waveguide spacing which is needed to reduce the crosstalk of the thermooptic heaters which are processed afterwards. A total of 16 waveguides were used in the S-shape, bringing the number of resolvable spots in the far field to 16 . Increasing this number will further decrease the beam width and will allow more accurate steering. After the S-shape, the waveguides taper to a $4 \mu \mathrm{m}$ width on which a 70nm deep grating is etched with a period of $630 \mathrm{~nm}$ and a duty cycle of 0.5 . The gratings are separated $5 \mu \mathrm{m}$.
Afterward a BCB (benzocyclobutene) layer of about 800nm is spun on top of the component on which $2.5 \mu \mathrm{m}$ wide heaters are processed using contact mask lithography. Each heater has a total length of $450 \mu \mathrm{m}$. These heaters are used to tune the phases of the different elements. They consist of a $100 \mathrm{~nm} \mathrm{Ti}$ layer and a $20 \mathrm{~nm} \mathrm{Au}$ layer to avoid oxidation of the heater. In a final stage, a $500 \mathrm{~nm}$ gold layer is processed on the contact pads of the heaters. A cross-section of the S-shape on which the heaters are processed is shown in Fig. 2(a).

The input waveguides shown in Fig. 1 are connected with a grating coupler which is optimized for near vertical coupling of infrared light from a fiber to the fundamental TE-like mode of an integrated circuit [11]. These are used to excite our structure. For the remainder of this paper, we assume to work only with TE-like light.

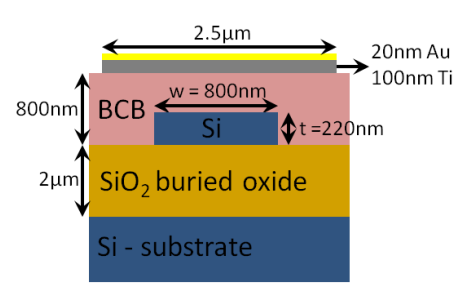

(a)

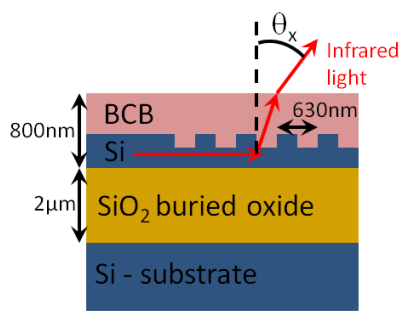

(b)
Fig. 2. Cross-sectional view of the (a) thermo-optic phase tuners, (b) grating coupler.

To split the light into 16 waveguides, two approaches can be taken: using an MMI (MultiMode Interference) splitter, or using a star coupler. When using an MMI-splitter, all waveguides will have an equal amount of power. This will result in an increased sidelobe level in the far-field. By making use of a star coupler, a natural apodization of the field over the OPA is obtained, and the simulation shows a sidelobe level decrease of $13 \mathrm{~dB}$ as can be seen in Fig. 3. In this Figure the simulated far-field of the beam steering component in the $\theta_{y^{-}}$ direction is shown for the two different cases at a wavelength of $1550 \mathrm{~nm}$. The FWHM (full-width-half-maximum) beam width becomes a bit larger and increases from $0.98^{\circ}$ to $1.26^{\circ}$.

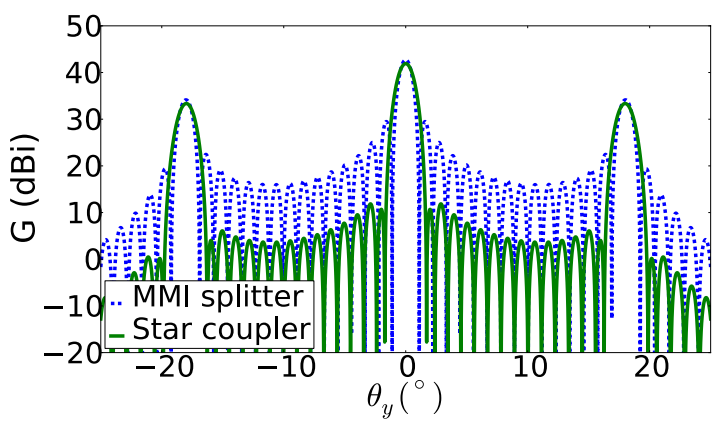

Fig. 3. Simulated far-field of a 16 element 1D OPA with equal amount of power in each element (MMI splitter) or with apodization (star coupler). A sidelobe reduction of $13 \mathrm{~dB}$ can be seen.

By using thermo-optic phase tuners, the beam can be shaped and steered in the $\theta_{y}$-direction. Silicon has a high thermooptic coefficient $\left(\partial n / \partial T=1.84 \times 10^{-4} \mathrm{~K}^{-1}\right)$ and different 
designs have been investigated in literature to optimize their performance [5], [12]. Here a simple heater design was used, which was proven to be sufficient to demonstrate the steering and focusing capabilities. The power needed for a $\pi$ phase shift at $1550 \mathrm{~nm}$ was measured to be around $P_{\pi}=15-20 \mathrm{~mW}$. This can be reduced by more elegant heater designs, such as insulating trenches. Different phase tuning approaches have been reported on silicon such as the use of liquid crystals [13], carrier depletion [14] or more recently using a NEMS (NanoElectroMechanical Systems) approach [15] which all require a small amount of power as they work capacitively. Depending on the wanted speed and performance, these can be integrated in the current design. Our current heaters have a speed which is limited to about $20 \mathrm{kHz}$, with a $10 \%-90 \%$ rise and fall time of $12.5 \mu \mathrm{s}$ and $7.2 \mu \mathrm{s}$, respectively. This is shown in Fig. 4, where the heater performance is measured in a balanced MachZehnder interferometer (MZI) where one arm is heated. The heater is driven by a $20 \mathrm{kHz}$ voltage signal for which the peak voltage corresponds to a $\pi$ phase shift.

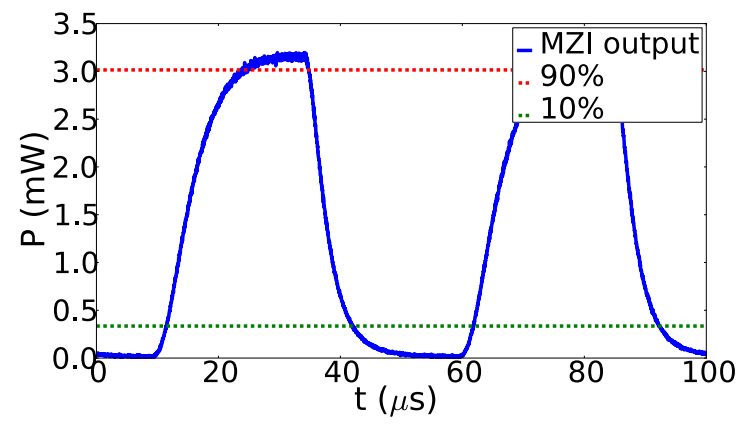

Fig. 4. Dynamic behavior of the heater. The output of a balanced MachZehnder interferometer is shown where one arm is heated. The heater is driven by a $20 \mathrm{kHz}$ voltage signal which gives a $\pi$ phase shift at the peak voltage.

While there are a lot of degrees of freedom in the $\theta_{y}$ direction, there is limited freedom in the $\theta_{x}$-direction. The beam characteristics in this direction are determined by the out-coupling grating. This is a second order grating which will send the light off-chip at an angle given by the grating equation:

$$
\sin \theta_{x}=\frac{\Lambda_{g r} n_{e f f, g r}-\lambda}{n_{c t} \Lambda_{g r}},
$$

with $\Lambda_{g r}$ the period of the grating $\left(\Lambda_{g r}=630 \mathrm{~nm}\right), \lambda$ the freespace wavelength, $n_{e f f, g r}$ the effective index of the guided mode in the grating area and $n_{c t}$ the refractive index of the background which is air $\left(n_{c t}=1\right)$ in this case. Using wavelength tuning, the beam can be steered in the $\theta_{x}$-direction. A cross-sectional view of the grating coupler is shown in Fig. 2(b).

\section{MEASUREMENT SETUP}

The far-field characteristics of the beam steering component are investigated using a Fourier imaging setup. The far-field is imaged on the back-focal plane of a high numerical aperture $(\mathrm{NA}=0.5)$ microscope objective (MO). This image is brought back to an infrared camera using two lenses as shown in
Fig. 5 [16]. Every point in the Fourier plane corresponds to a specific direction of emission and thus represents the far-field of the beam steering component. Light from a tunable laser is sent through a polarization controller and is then coupled into the beam steering component using a grating coupler for near-vertical coupling. The radiated light is then imaged using the Fourier imaging setup. A probe card is placed in front of the sample to drive the different heaters.

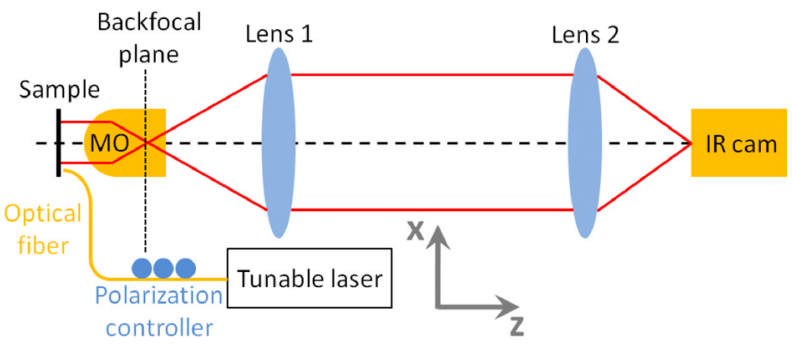

Fig. 5. A Fourier imaging setup is used to study the far-field characteristics of the beam steering component.

The focusing capabilities of the component are studied with the setup shown in Fig. 6. The structure is again excited using a tunable laser and a polarization controller. At a certain distance $f$, a single mode optical fiber is placed to capture the light radiated from the structure. The sample is positioned at a certain angle $\theta_{x}$ which is the out-coupling angle of the grating. The light is then focused in the yz-plane on the tip of the fiber in one-dimension and the received power is measured.

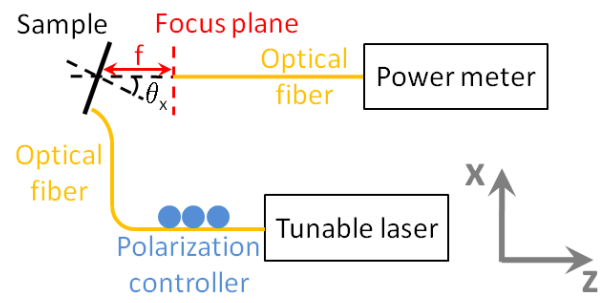

Fig. 6. Using a fiber at a distance $f$ of the sample, the focusing capability of the structure is investigated.

\section{Measurement Results And discussion}

First we discuss the far-field pattern of the beam steering component. Next, we investigate the wavelength and thermooptic steering behavior. Then we look into the focusing capabilities of the OPA. Finally a short discussion is given on the power efficiency of the OPA.

\section{A. Far-field pattern}

In Fig. 7, the measured far-field of the beam steering component is shown at a wavelength of $1550 \mathrm{~nm}$. A broad beam in the $\theta_{x}$-direction can be seen, which is determined by the out-coupling grating and centered around $\theta_{x}=10^{\circ}$ being the out-coupling angle of the grating given by Eq. (1). In the $\theta_{y}$-direction, a typical OPA far-field pattern can be seen. The beam is narrow and the different output orders are visible with a spacing of $18^{\circ}$. This spacing can be increased by 
decreasing the waveguide spacing. For $450 \mathrm{~nm}$ wide integrated waveguides on SOI operating at a wavelength of $1.55 \mu \mathrm{m}$, the minimal center-to-center spacing is about $1.3 \mu \mathrm{m}$ to avoid optical coupling between the waveguides. In the next sections, we will investigate cross-sectional views of the far-field of the beam steering component to study the wavelength steering and thermo-optic steering and shaping behavior.

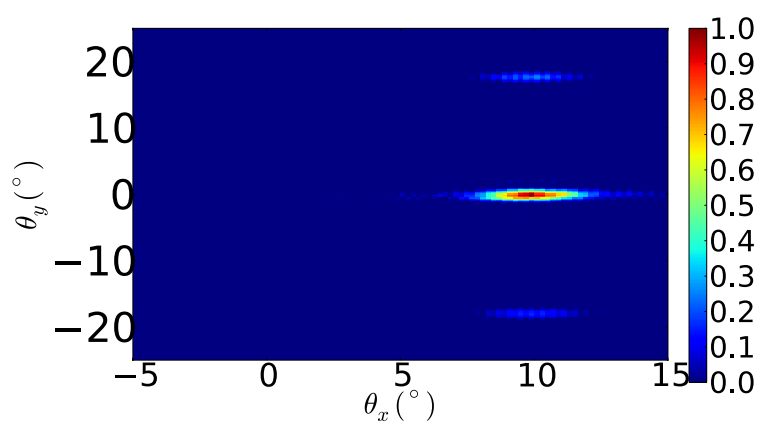

Fig. 7. Measured far-field pattern of the beam steering component at a wavelength of $1550 \mathrm{~nm}$.

\section{B. Wavelength steering}

By tuning the wavelength, the beam shifts in the $\theta_{x^{-}}$ direction. Fig. 8 shows the far-field pattern along the $\theta_{x}$ direction for three different wavelengths. The FWHM beam width is measured to be $3.0^{\circ}, 2.8^{\circ}$ and $1.6^{\circ}$ for a wavelength of $1520 \mathrm{~nm}, 1550 \mathrm{~nm}$ and $1580 \mathrm{~nm}$, respectively and the beam is steered over a $6.7^{\circ}$ range for a $60 \mathrm{~nm}$ wavelength shift.

As the field that leaves a grating coupler with a fixed grating period is an exponential decaying field, the far-field pattern does not look very nice as can be seen in Fig. 8. The smaller beam width for increasing wavelength can be explained due to the fact that the grating couples the light out less efficiently, so that the exponential tail becomes larger and the far-field beam width will decrease. The wavelength dependent steering can simply be removed by cleaving the chip and using the end facet of the waveguides as radiating elements which is the approach taken in [5].

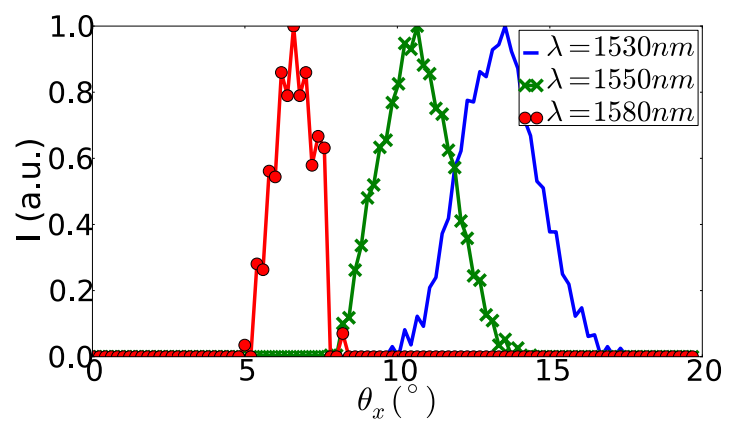

Fig. 8. Measured far-field in the $\theta_{x}$-direction using wavelength steering.

\section{Fixed beam steering}

By using a different input waveguide of the star coupler as shown in Fig. 1, fixed steering of the beam in the $\theta_{y}$-direction is observed. The free spectral range of the far-field in the $\theta_{y}$ direction is equal to $\arcsin \left(\lambda / \Lambda_{y}\right)$ with $\lambda$ the wavelength and $\Lambda_{y}$ the spacing of the grating couplers $\left(\Lambda_{y}=5 \mu \mathrm{m}\right)$. At $\lambda=$ $1.55 \mu \mathrm{m}$, this corresponds to $18^{\circ}$. We have chosen 9 access waveguides and each waveguide results in a shift of $2^{\circ}$ so that the complete FOV is covered. A cross-sectional view of the far-field in the $\theta_{y}$-direction for different access waveguides is shown in Fig. 9. In this Figure, only the far-fields for 5 input waveguides are plotted in order not to overload the Figure. The envelope of the far-field pattern is shown as well and has a FWHM of $23^{\circ}$ which is determined by the width of the grating couplers. The beam width is $1.27^{\circ}$ which compares well to the simulated value of $1.26^{\circ}$. The different output orders at a spacing of $18^{\circ}$ are visible as well.

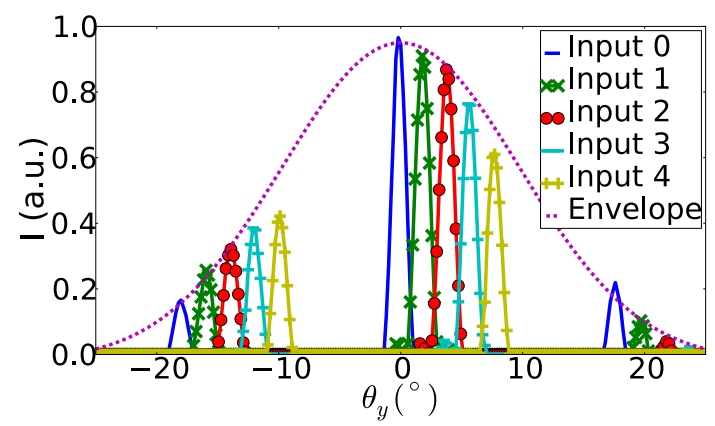

Fig. 9. Measured far-field in the $\theta_{y}$-direction for different input waveguides (Fig. 1) at $1550 \mathrm{~nm}$. The dashed line shows the envelope of the far-field pattern.

\section{Thermo-optic beam steering}

By using the thermo-optic phase tuners, we can now steer the beam over the complete FOV in the $\theta_{y}$-direction. As the phase change $\phi$ is directly proportional to the dissipated power in the heater, and the latter is proportional to the applied voltage $V$ squared, we use the simple model:

$$
\phi=\beta V^{2}
$$

Where $\beta$ is a fixed parameter. Using this simple model, the $2 \pi$ phase resets can be taken into account. To steer the beam at an angle $\theta_{y}$, the phase difference $\Delta \phi$ between each waveguide is equal to:

$$
\Delta \phi=\frac{2 \pi \Lambda_{y}}{\lambda} \sin \theta_{y}
$$

In Fig. 10, a cross-sectional view of the far-field in the $\theta_{y^{-}}$ direction is shown when the beam is steered at different angles. When steering at an $\theta_{y}$-angle of $18^{\circ}$, the far-field coincides with the original far-field at $0^{\circ}$ which proves the validity of our simple model.

\section{E. Beam focusing}

By programming the phase tuners as to act as a lens, light is focused above the chip in one-dimension. Using a scalar approach, we simulated the coupling efficiency from the OPA to the fiber as a function of the distance $f$ between the chip and the fiber (Fig. 6) as shown in Fig. 11. For very small $f$, 


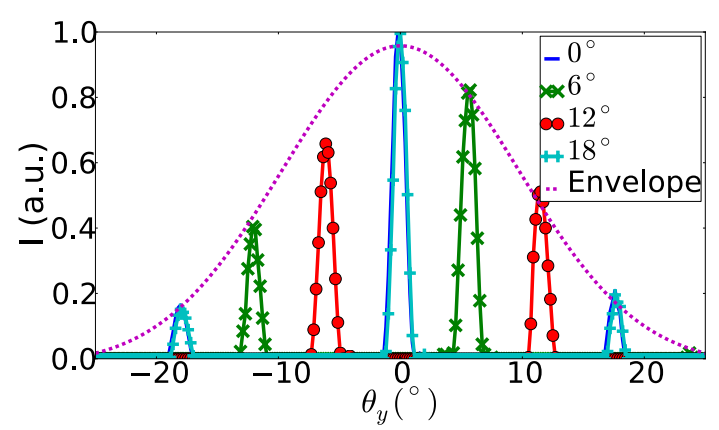

Fig. 10. Measured far-field in the $\theta_{y}$-direction using thermo-optic steering (Fig. 1) at $1550 \mathrm{~nm}$. The beam is steered to the angles $0^{\circ}, 6^{\circ}, 12^{\circ}$ and $18^{\circ}$. The dashed line shown the envelope of the far-field pattern.

the NA of the fiber is too small to capture this light and a low efficiency is seen. At a distance of $0.33 \mathrm{~mm}$, an optimum is found, when the NA of the component matches the one of the optical fiber. Note that this loss of $2 \mathrm{~dB}$ is only the loss in one dimension and is due to the fill-factor of the OPA.

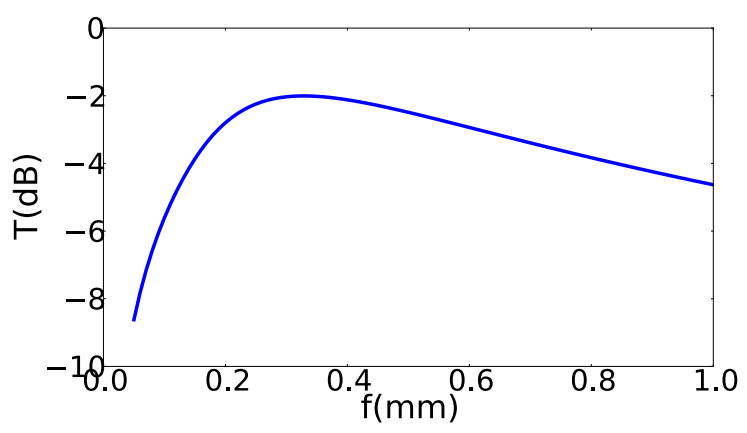

Fig. 11. One-dimensional simulated coupling efficiency of the 16 element OPA to an optical fiber as a function of distance between the OPA and the fiber at a wavelength of $1550 \mathrm{~nm}$. For each point, the phases are set to focus the light onto the fiber.

In Fig. 12 the measured power into the fiber is given for the case when the light is focused onto the fiber and when no focusing is used at a wavelength of $1550 \mathrm{~nm}$. The power shown is relative to the power received when the fiber is exactly on top of the structure $(f=0 \mathrm{~mm})$ and no focusing is used. At first instance, a simple model is used to focus the light, as explained above. Afterward a rough optimization run did not show any significant improvement. There is an optimum focus point at $f=0.4 \mathrm{~mm}$, which shows the focusing capabilities of the $1 \mathrm{D}$ OPA. An increase of $3 \mathrm{~dB}$ in received power is observed. By using an apodized grating, light can also be focused in the other dimension at a fixed point.

\section{F. Power budget}

The out-coupling efficiency of the $4 \mu \mathrm{m}$ wide waveguides has been investigated in 2D using the eigenmode expansion tool CAMFR (CAvity Modelling FRamework, http://camfr. sourceforge.net/). The out-coupling efficiency is about 38$40 \%$ over a $100 \mathrm{~nm}$ wavelength range around $1550 \mathrm{~nm}$, but this can be easily increased to over $70-80 \%$ using a silicon

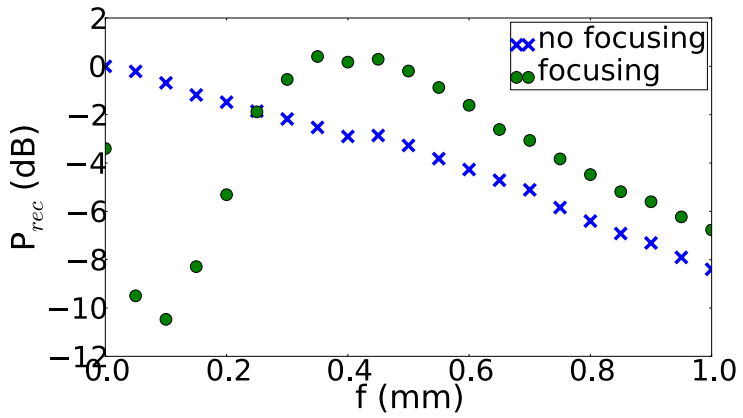

Fig. 12. Measured relative received power into an optical fiber at a distance $f$ of the 16 element OPA at a wavelength of $1550 \mathrm{~nm}$. For each point, the phases are set to focus to light onto the fiber. The power is relative to the power received at $f=0 \mathrm{~mm}$ when no focusing is used.

overlay [17]. The loss of the star coupler is less than $1.5 \mathrm{~dB}$. The waveguide loss is about $3 \mathrm{~dB} / \mathrm{cm}$, bringing the total waveguide losses in the component to only $0.2 \mathrm{~dB}$

\section{CONCLUSION}

We have shown a 16 element one-dimensional optical phased array on silicon-on-insulator. Using a star coupler to split the light into 16 waveguides, the sidelobe level is reduced by $13 \mathrm{~dB}$. The star coupler further allows us to demonstrate fixed beam steering by changing the input waveguide. The field-of-view was $23^{\circ}$ using $4 \mu \mathrm{m}$ wide grating couplers spaced $5 \mu \mathrm{m}$. The different output orders of the array were spaced $18^{\circ}$. This field-of-view and output order spacing could easily be increased by decreasing the waveguide width and spacing, respectively, but will result in a broader beam as the number of elements stays constant. The beamwidth was $1.27^{\circ}$. Using thermo-optic phase tuners, the beam was steered over its complete field-of-view. By programming the phase tuners as a lens, focusing capabilities have been shown as well.

\section{ACKNOWLEDGMENT}

The authors would like to thank Steven Verstuyft and Elewout Hallynck for help in the post-processing of the component.

\section{REFERENCES}

[1] D. A. B. Miller, "Device Requirements for Optical Interconnects to Silicon Chips," Proceedings of the IEEE, vol. 97, no. 7, pp. 1166-1185, 2009.

[2] P. F. McManamon, P. J. Bos, M. J. Escuti, J. Heikenfeld, S. Serati, H. K. Xie, and E. A. Watson, "A Review of Phased Array Steering for NarrowBand Electrooptical Systems," Proceedings of the IEEE, vol. 97, no. 6, pp. 1078-1096, 2009.

[3] K. Van Acoleyen, W. Bogaerts, J. Jágerská, N. Le Thomas, R. Houdré, and R. Baets, "Off-chip beam steering with a one-dimensional optical phased array on silicon-on-insulator," Optics Letters, vol. 34, no. 9, pp. 1477-1479, 2009.

[4] K. Van Acoleyen, H. Rogier, and R. Baets, "Two-dimensional optical phased array antenna on silicon-on-insulator," Optics Express, vol. 18, no. 13, pp. 13655-13660, 2010.

[5] D. N. Kwong, Z. Yang, A. Hosseini, and R. T. Chen, "Integrated optical phased array based large angle beam steering system fabricated on silicon-on-insulator," Proceedings of the SPIE - The International Society for Optical Engineering, vol. 7943, pp. 79430Y-1 - 79430Y-6, 2011. 
[6] K. Van Acoleyen, W. Bogaerts, and R. Baets, "Two-Dimensional Dispersive Off-Chip Beam Scanner Fabricated on Silicon-On-Insulator," IEEE Photonics Technology Letters, vol. 23, no. 17, pp. 1270-1272, 2011.

[7] Y. Li, S. Meersman, and R. Baets, "Realization of fiber based laser Doppler vibrometer with serrodyne frequency shifting," Applied Optics, to be published, vol. 50, no. 17, pp. 2809-2814, 2011.

[8] G. Yurtsever, P. Dumon, W. Bogaerts, and R. Baets, "Integrated photonic circuit in silicon on insulator for Fourier domain optical coherence tomography," in Proceedings of SPIE-The International Society for Optical Engineering, vol. 7554, 2010.

[9] S. K. Selvaraja, P. Jaenen, W. Bogaerts, D. Van Thourhout, P. Dumon, and R. Baets, "Fabrication of Photonic Wire and Crystal Circuits in Silicon-on-Insulator Using 193-nm Optical Lithography," Journal of Lightwave Technology, vol. 27, no. 18, pp. 4076-4083, 2009.

[10] P. Dumon, W. Bogaerts, D. Van Thourhout, D. Taillaert, R. Baets, J. Wouters, S. Beckx, and P. Jaenen, "Compact wavelength router based on a silicon-on-insulator arrayed waveguide grating pigtailed to a fiber array," Optics Express, vol. 14, no. 2, 2006.

[11] G. Roelkens, D. Vermeulen, D. Van Thourhout, R. Baets, S. Brision, P. Lyan, P. Gautier, and J. M. Fedeli, "High efficiency diffractive grating couplers for interfacing a single mode optical fiber with a nanophotonic silicon-on-insulator waveguide circuit," Applied Physics Letters, vol. 92 , no. 13, p. 131101, 2008.

[12] J. Van Campenhout, W. M. J. Green, S. Assefa, and Y. A. Vlasov, "Integrated NiSi waveguide heaters for CMOS-compatible silicon thermooptic devices," Optics Letters, vol. 35, no. 7, pp. 1013-1015, 2010.

[13] W. De Cort, J. Beeckman, R. James, F. A. Fernandez, R. Baets, and K. Neyts, "Tuning silicon-on-insulator ring resonators with in-plane switching liquid crystals," Journal of the Optical Society of America B - Optical Physics, vol. 28, no. 1, pp. 79-85, 2011.

[14] N. N. Feng, S. R. Liao, D. Z. Feng, P. Dong, D. W. Zheng, H. Liang, R. Shafiiha, G. L. Li, J. E. Cunningham, A. V. Krishnamoorthy, and M. Asghari, "High speed carrier-depletion modulators with $1.4 \mathrm{~V}-\mathrm{cm} \mathrm{V}$ pi L integrated on $0.25 \mathrm{mu} \mathrm{m}$ silicon-on-insulator waveguides," Optics Express, vol. 18, no. 8, pp. 7994-7999, 2010.

[15] K. Van Acoleyen, J. Roels, T. Claes, D. Van Thourhout, and R. Baets, "NEMS-based optical phase modulator fabricated on Silicon-OnInsulator," in 8th International Conference on Group IV Photonics (GFP 2011), Londen, UK, 2011, pp. FC6, 371-373.

[16] N. Le Thomas, R. Houdré, M. V. Kotlyar, D. O'Brien, and T. E. Krauss, "Exploring light propagating in photonic crystals with Fourier optics," Journal of the Optical Society of America B, vol. 24, no. 12, pp. 29642971, 2007.

[17] D. Vermeulen, S. Selvaraja, G. Verheyen, P. Lepage, W. Bogaerts, and G. Roelkens, "High-efficiency Silicon-On-Insulator Fiber-to-Chip Grating Couplers Using a Silicon Overlay," in 6th International Conference on Group IV Photonics (GFP 2009), United States, 2009, p. FPd1.

Karel Van Acoleyen (M'10) received the Erasmus Mundus Master of Science in Photonics degree in 2008 from Ghent University, Belgium and the Royal Institute of Technology (KTH), Stockholm, Sweden. Since 2008 he is working towards a PhD in the Photonics Research Group of Ghent University-imec. His research interests include integrated photonics and its applications in beam steering, scanning and free-space sensing. He is a member of IEEE Photonics Society.

Katarzyna Komorowska received M.Sc. degree in Physics (Applied Optics) in 1999 and Ph.D. in Chemistry in 2003 from Wroclaw University of Technology in Poland. From 2003 to 2006 she was a Postdoctoral Researcher with Ecole Normale Supérieure de Cachan in France in Quantum and Molecular Photonics Laboratory (LPQM). Since 2006 she has been Postdoctoral Researcher with the Photonics Research Group, Department of Information Technology, Ghent University in Belgium. Her current research interests include heterogeneous integration and photonic devices fabrication technologies.
Wim Bogaerts is professor in Silicon Photonics in the Photonics Research Group at Ghent University. He completed his studies in engineering (applied physics) at Ghent University in 1998 and joined the department of information technology (INTEC) at both Ghent University and the imec nanotechnology research center. He received his $\mathrm{PhD}$ degree in 2004. His research focuses on the modeling, design and fabrication of silicon photonic components and circuits, with a strong focus on large-scale integration. He also coordinates the joint work in silicon photonics between Ghent University and imec. He is a member of the IEEE Photonics Society, the Optical Society of America (OSA) and SPIE.

Roel Baets (M'88 - SM'96) is full professor at Ghent University (UGent). He is also associated with imec. He has management responsibilities within the Photonics Research Group of UGent, the Center for Nano- and Biophotonics (NB Photonics) of UGent, the international Erasmus Mundus M.Sc. program in Photonics and the joint UGent-imec research program on silicon photonics. Roel Baets received an M.Sc. degree in Electrical Engineering from Ghent University in 1980 and a second M.Sc. degree from Stanford University in 1981. He received a PhD degree from Ghent University in 1984. From 1984 till 1989 he held a postdoctoral position at imec (with detachment to Ghent University). Since 1989 he has been a professor in the Engineering Faculty of UGent where he founded the Photonics Research Group. From 1990 till 1994 he has also been a part-time professor at the Technical University of Delft and from 2004 till 2008 at the Technical University of Eindhoven. Roel Baets has mainly worked in the field of integrated photonic components. He has made contributions to research on semiconductor laser diodes, guided wave and grating devices and to the design and fabrication of photonic ICs, both in III$\mathrm{V}$ semiconductors and in silicon. As part of a team of 6 professors he leads the Photonics Research Group at UGent. With about 60 researchers this group is involved in numerous national and international research programs. The silicon photonics activities of the group are part of a joint research initiative with imec. Roel Baets is also director of the multidisciplinary Center for Nano- and Biophotonics (NB Photonics) at UGent, founded in 2010. Roel Baets was co-founder of the interuniversity UGent-VUB M.Sc. programme in Photonics and of the international Erasmus Mundus M.Sc. programme in Photonics, of which he chairs the Board. Roel Baets is a grant holder of the Methusalem programme of the Flemish government and of the European Research Council (ERC). He is a Fellow of the IEEE. 